\title{
Perfil y diferencias antropométricas y físicas de gimnastas de tecnificación de las modalidades de artística y rítmica \\ Anthropometric and physical differences of the gymnasts from the talent identification program of the artistic and rhythmic specialties
}

\author{
*Marta Leyton Román, *Vicente Luis del Campo, **Rafael Sabido Solana y *Jesús Morenas Martín \\ *Universidad de Extremadura, **Universidad Miguel Hernández. Elche
}

\begin{abstract}
Resumen: El objetivo del trabajo fue describir y comparar las características físicas y antropométricas de 25 gimnastas pertenecientes al grupo de tecnificación de la Federación Extremeña de Gimnasia. Estas gimnastas fueron clasificadas según modalidad gimnástica: artística femenina $(\mathrm{GAF})(\mathrm{n}=13)$ y gimnasia rítmica (GR) $(\mathrm{n}=12)$. Las variables dependientes incluyen valoraciones del porcentaje graso, a través de una báscula electrónica de columna con tallímetro $(S E C A 220 \mathrm{~cm})$; perímetros corporales, a través de un plicómetro (Holtain); diámetros y pruebas específicas de flexibilidad, a través de cinta métrica (CM 3m); frecuencia cardíaca, a través del test de Ruffier y uso de pulsómetro (Polar Fo); fuerza isométrica del tren inferior, donde se utilizó una célula de carga (SSMAJ 5000N), y la capacidad de salto, a través de una plataforma de contacto (Lafayette CVP A73). Los resultados concluyen que GAF obtiene mejor resultado en las pruebas de flexibilidad $(p<.05)$. Además, el grupo GR cuanto menor porcentaje graso posee mayor capacidad de salto SJ (r=-.774; $p<.01)$ y CMJ $(\mathrm{r}=-.600 ; p<.05)$. En cambio, el grupo GAF cuanto mayor índice de masa corporal menor es su flexibilidad $(p<.01)$. Se concluye que existen diferencias en composición corporal y pruebas físicas entre las modalidades gimnásticas femeninas de artística y rítmica, además de encontrar relaciones entre el rendimiento de dichas pruebas y variables antropométricas.
\end{abstract}

Palabra clave: gimnasia, características antropométricas, flexibilidad, fuerza isométrica, salto.

Abstract: The aim of our research was to describe the physical and anthropometric characteristics of 25 gymnasts from the talent identification program of the Extremeña Gymnastics Federation. Subjects were classified according to their specialty: women's artistic gymnastic (WAG) and rhythmic gymnastics (RG). The dependent variables include body fat percentage through an electronic scale column with stadiometer (SECA $220 \mathrm{~cm})$; body circumferences through a plicometer (Holtain); body diameters and specific tests of flexibility through a tape (CM 3m); a heart rate through Ruffier test and a heart rate monitor (Polar FO); isometric strength of lower extremities through a load cell (SSMAJ 5000N); and jumping ability through a contact mat (Lafayette CVP A73). The results concluded that the WAG group got the best results in flexibility tests ( $p<.05)$. Also, the RG group had lower body fat percentage and greater SJ jump ability $(\mathrm{r}=-.774 ; p<.01)$ and $\mathrm{CMJ}(\mathrm{r}=-.600 ; p<.05)$. However, the WAG group showed a negative relation between body mass index and flexibility $(p<.01)$. We conclude that there are differences in body composition and physical tests between the specialties/styles of women's artistic gymnastics and female rhythmic gymnastics; we also found relationships between the performance of such tests and anthropometric variables.

Key words: gymnastics, anthropometric characteristics, flexibility, isometric strength, jump.

\section{Introducción}

La gimnasia femenina es una disciplina deportiva que requiere de un duro, prolongado y constante entrenamiento físico a lo largo de toda la carrera deportiva. Sin embargo, una gimnasta de alto nivel no sólo necesita poseer una serie de aptitudes físicas, sino que sus características antropométricas son además muy importantes, y en ocasiones, determinantes en la victoria de la gimnasta ya que le proporciona las características biomecánicas necesarias para la ejecución de ejercicios gimnásticos (Díaz, Mauri, García \& Jiménez, 2008). Entre ellas, la composición corporal es un determinante importante en el óptimo rendimiento de las gimnastas. De esta manera, el incremento de la grasa corporal rara vez conduce a altos niveles de rendimiento ya que determinaría una reducción en el índice de fuerza relativa al peso (Sands, Caine \& Borms, 2003).

Una característica de la gimnasia rítmica en comparación con la gimnasia artística es que la mayoría de las gimnastas son altas, con tronco y caderas estrechas y muy delgadas (Mendizábal, 2001). Sin embargo, en la gimnasia artística, donde el factor velocidad juega un papel fundamental dentro de la estructura espacial de los mismos, se necesita una temprana especialización y un tipo de gimnastas de reducidas dimensiones corporales (López, Vernetta \& De la Cruz, 1993).

No es un secreto que las gimnastas que llegan a la élite, han pasado por un altísimo proceso selectivo y la mayoría de ellas son genéticamente delgadas antes de iniciarse en la gimnasia (Mendizábal, 2001). Un bajo porcentaje de grasa es beneficioso en la gimnasia, sobre todo en la

Fecha recepción: 06-07-11 - Fecha envío revisores: 18-07-11 - Fecha de aceptación: 21-11-11 Correspondencia: Marta Leyton Román

Avda. del Perú $\mathrm{n}^{\circ} 17,9^{\circ} \mathrm{B}$,

06011 Badajoz

E-mail: martabudy@hotmail.com gimnasia artística donde el peso del cuerpo es impulsado en contra de la fuerza de la gravedad en multitud de elementos. Sin embargo, se han descrito similares porcentajes de grasa corporal en gimnastas de diferentes niveles y en otras deportistas (Taaffe, Robinson, Snow \& Marcus, 1997; Deutz, Benardot, Martin \& Cody, 2000) siendo superiores éstos en gimnastas femeninas con respecto a sus homólogos masculinos (Weimann, 2002; Weimann, Witzel, Schwidergall \& Böhles, 2000). El 90\% de los gimnastas tanto de la modalidad de rítmica como de artística, se clasifica en un perfil ecto-mesomórfico (Irurtia, Busquets, Marina, Galilea \& Carrasco, 2009). Silva (2005), en un estudio con gimnastas prepúberes de las modalidades de rítmica y artística, determinó que el porcentaje graso medio de las gimnastas era de $16.1 \pm 4.38$, ya que, considerando que el peso corporal tiene influencia sobre la resistencia y los índices de fuerza, las gimnastas se revelan como elegantes por dar la imagen de figuras magras y de reducido peso (Weimann, 2000).

Además de las características antropométricas, dentro de las cualidades específicas de la gimnasia, es imprescindible hablar de la fuerza. En concreto, el incremento de la fuerza relativa incrementaría la facilidad del movimiento requerido para el éxito en este deporte tipificado por movimientos contra la fuerza de la gravedad. De este modo, son primordiales para el rendimiento óptimo de las gimnastas los altos índices de fuerza y de potencia relativas al peso corporal (Sands et al., 2003). Por este motivo, en las modalidades gimnásticas destaca la fuerza explosiva, que en el caso de la gimnasia rítmica se demuestra en elementos como las zancadas, y el caso de la gimnasia artística resulta muy importante en elementos como saltos y mortales, sobre todo en la barra de equilibrios.

La valoración de la fuerza explosiva se puede determinar mediante tests dinámicos e isométricos. Entre las valoraciones dinámicas destacan los distintos tests de salto. La valoración de la capacidad de salto ha sido utilizada frecuentemente en modalidades gimnásticas dado que 
constituyen una acción motriz esencial en la actividad gimnástica, tanto en aquellas disciplinas que poseen un carácter acrobático como en aquellas que no lo tienen (Morenilla, Sierra, Bueno \& Rodríguez, 2001). Trabajos como el de Bradshaw y Le Rossignol (2004) y León (2006) apuntan que unos mejores resultados en salto tipo squat jump (SJ) y countermovement jump (CMJ) están relacionados con unos mejores resultados en suelo, en el caso de la gimnasia artística.

Grande, Figueroa, Hontoria y Bautista (2009) analizaron y compararon la evolución de la capacidad de salto de los equipos nacionales españoles de gimnasia artística femenina $(n=5)$ y Gimnasia Rítmica ( $\mathrm{n}=11)$, durante la preparación del Campeonato del Mundo (2007). En los resultados se observaron valores medios superiores en las alturas de salto de las gimnastas de gimnasia artística femenina, tanto en elSJ como en elCMJ, aunque las diferencias no fueron estadísticamente significativas.

La fuerza explosiva valorada en condiciones isométricas tiene relación con el análisis de la curva fuerza-tiempo, concretamente con el mayor valor de la pendiente, donde se produce el mayor incremento de la tensión muscular (manifestación de la fuerza) por unidad de tiempo. Se corresponde con el máximo índice de manifestación de la fuerza (MIMFmax), que está en relación, a su vez, con la habilidad del sistema neuromuscular para desarrollar una alta velocidad de acción o para crear una fuerte aceleración en la expresión de fuerza (González \& Gorostiaga, 2002). Sin embargo, es importante tener en cuenta que la fatiga muscular influye sobre la fuerza explosiva aplicada en el salto (Márquez, Orihuelo, Jiménez \& Fernández, 2005).

La flexibilidad es otra cualidad física importante para determinar el rendimiento de un gimnasta ya que permite a los deportistas realizar de manera óptima los diferentes elementos gimnásticos, ya sean dinámicos o estáticos (Ariza, 2004). De hecho, Sands et al. (2003), afirman que los resultados obtenidos en gimnasia tanto artística como rítmica, a menudo son influenciados directamente por la capacidad del gimnasta de alcanzar ciertas posiciones del cuerpo a través de la flexibilidad.

En síntesis, la aportación principal del trabajo contextualizado en el deporte de la gimnasia y una vez conocidas las principales variables físicas que determinan el rendimiento de dicho deporte, se centra en:

a)Conocer las diferencias existentes, así como las particularidades propias de cada modalidad gimnástica (artística femenina y rítmica) en cuanto a características antropométricas, flexibilidad, capacidad de salto y producción de fuerza rápida en condiciones isométricas. b)Determinar la relación entre diferentes capacidades en los grupos de gimnastas evaluados.

\section{Método}

\subsection{Participantes}

La muestra del estudio estuvo conformada por 25 gimnastas pertenecientes al plan de detección de talentos de la Federación Extremeña de Gimnasia, 12 de ellas pertenecían a la especialidad de gimnasia rítmica con edad media de $11.33 \pm 1.15$ años, masa media de $36.29 \pm 7.67 \mathrm{~kg}$ y altura media de $1.45 \pm .11 \mathrm{~m}$; y 13 pertenecían a la especialidad de gimnasia artística femenina con una edad media de $9.69 \pm 1.84$ años, masa media de $30.19 \pm 9.10 \mathrm{~kg}$ y altura media de $1.38 \pm .19 \mathrm{~m}$.

Los padres de los participantes, previo comienzo de la investigación, fueron informados de los objetivos y del proceso de investigación, consintiendo y aceptando libremente la participación de sus hijos en el estudio. Se informó a los participantes sobre la finalidad del estudio y se proporcionó por escrito las instrucciones a cumplir durante el protocolo de medición.

\subsection{Variables}

\subsubsection{Variables dependientes}

- Porcentaje graso (\% graso).

- IMC: Índice de Masa Corporal $\left(\mathrm{kg} / \mathrm{m}^{2}\right)$.

- Valor conseguido respecto a pliegues (cm) del bíceps, tríceps, axilar, pecho, subescapular, suprailiaco, abdominal, muslo y gemelo; circunferencias $(\mathrm{cm})$ del brazo, pecho, cintura, cadera, muslo y gemelo; y diámetros $(\mathrm{cm})$ del codo y de la rodilla.
-Altura en centímetros (cm) conseguida en los saltos: squat jump (SJ), countermovement jump (CMJ), drop jump (DJ) partiendo desde una altura del $40 \mathrm{~cm}$ y repeat jump (R5).

-Fuerza isométrica de los extensores del tobillo. Respecto a la curva fuerza-tiempo en situación isométrica se consideraron las variables: Pico máximo de fuerza producido (Pmax.), expresado en Newton (N); y Máximo índice de manifestación de la fuerza (MIMFmax), que indica el mayor valor en la pendiente de la curva fuerza-tiempo, expresado en Newton/milisegundos ( $/ \mathrm{ms}$.);

-Valor de la frecuencia cardiaca $\left(l a t / \mathrm{min}^{-1}\right)$ a través del Test de Ruffier, donde se determinaron las pulsaciones ( $\mathrm{n}^{\circ} \mathrm{pul}$ ) en reposo, tras el ejercicio y tras 1 minuto.

-Prueba de fondos $\left(\mathrm{n}^{\circ}\right)$ en 30 segundos.

-Flexibilidad (cm) en extensión lumbar, hombros, isquiotibiales y spagat.

La concreción de las variables respecto a los valores antropométricos así como de los test de salto se realizó siguiendo a León (2006) mientras que las variables flexibilidad se concretaron según García, Navarro y Navarro (2009).

\subsubsection{Variables Independientes}

La variable independiente principal fue el tipo de modalidad gimnástica, con dos niveles: Gimnasia Artística Femenina (GAF) y Gimnasia Rítmica (GR).

\subsection{Procedimiento de medición y materiales}

Todas las mediciones fueron realizadas en el Laboratorio de Control y Aprendizaje Motor, de la Facultad de Ciencias del Deporte, de la Universidad de Extremadura. Las medidas fueron tomadas por Licenciados en Ciencias del Deporte, con amplia experiencia en el instrumental utilizado, y bajo la supervisión de entrenadores y jueces de gimnasia con experiencia en el entrenamiento y competición de gimnasia femenina.

Antes de realizar todas las pruebas, se le permitió al participante realizar un ensayo de calentamiento en cada prueba, para que se familiarizase con el instrumental y las mediciones. Todas las mediciones se realizaban en el mismo día, y en el mismo orden que a continuación aparece, con el fin de controlar el orden de aplicación de las pruebas realizadas entre las gimnastas.

Entre las diferentes mediciones el participante tenía un periodo de descanso de 10 minutos, y después, antes de cada medición el participante realizaba un calentamiento específico relacionado con la medición que fuese a realizar, de otros 10 minutos de duración.

En primer lugar, se midieron los pliegues de tríceps, subescapular, suprailiaco, abdominal, del muslo ydel gemelo con un plicómetromodelo Holtain. Posteriormente se calculó el porcentaje graso a través de la siguiente fórmula (Yuhasz, 1974): Chicas \% graso: 4.56 + (suma 6 pliegues*.143)

Se optó por esta ecuación por su alta validez ( $\mathrm{r}=.84$; Katch \& McArdle, 1975); y por su practicidad, ya que brinda en un solo cálculo el porcentaje graso.

Después se midieron las circunferencias con una cinta métrica y los diámetros corporales con un calibre mecánico marca Schwyz, modelo SC141402. Antes de llevar a cabo las diferentes pruebas de fuerza y salto se les pedía a los participantes que realizasen un calentamiento de 10 minutos, que consistía básicamente en carrera continua, movilidad de todas las articulaciones, y saltos.

Para el análisis cinemático de los saltos se utilizó una plataforma de contacto, modelo Lafayette CVP 1723. Esta plataforma permite la medición de los tiempos de contacto durante los test de los diferentes saltos. Los saltos Squat Jump (SJ) Countermovement Jump (CMJ), Drop Jump (DJ) y Repeat Jump (R5) se realizaron siguiendo los protocolos establecido por Bosco (1994). En el SJ se controló el ángulo de las rodillas para mantener la flexión a $90^{\circ}$. La flexión de rodillas en el CMJ se dejó libre. En el SJ y el CMJ se trató de mantener el tronco lo mas vertical posible, se basaba en la realización de una ejecución máxima y las manos fijadas en las caderas para impedir la intervención de las 
extremidades superiores en los saltos. El salto DJ, consistió en una caída vertical libre desde una altura de $0.45 \mathrm{~m}$ sobre la plataforma (Grande, Sampedro, Rivilla-García, Bofill \& Hontoria, 2010). Desde la altura establecida se pide al gimnasta que dé un paso al frente a juntar pies, sin saltar, y caer verticalmente sobre la plataforma con los dos pies a la vez intentando amortiguar la caída. El salto R5, consiste en una sucesión de 5 saltos verticales. En él, se busca la máxima altura limitando en lo posible la deformación músculo-articular de la cadena cinética de salto, como consecuencia del violento contacto con el suelo después del vuelo precedente. La secuencia de realización fue CMJ, SJ, DJ y R5. Se realizó esta secuencia dos veces con 30 segundos de descanso entre cada salto. Para la realización de los tests las gimnastas utilizaban su calzado específico de gimnasia. En cuanto a los datos provenientes de las pruebas de salto, se obtuvo el mejor valor con respecto a la altura de cada tipo salto.

A continuación, tenía lugar el test de Ruffier, para comprobar la diferencia en un salto $\mathrm{CMJ}$ antes e inmediatamente después de una actividad de 30 sentadillas profundas. Durante este test se pedía a los deportistas que tocasen el suelo con las puntas de los dedos, flexionando para ello las rodillas, y no la cadera, en un máximo de 45 segundos, además se comprobaron las pulsaciones de los deportistas (con un pulsómetro marca Polar F6 con banda de pecho Wear Link) antes de la realización de las sentadillas, inmediatamente después y tras 1 minuto de reposo, para comprobar la evolución de la actividad.

Para el registro de la carga mecánica se utilizó un dinamómetro isométrico marca Interface, modelo SSM-AJ-5000N. Para la medición de la fuerza isométrica de los extensores del tobillo, se colocaba a la participante sentada con la espalda totalmente apoyada en la pared.

Tabla 1. Estadísticos descriptivos de las variables dependientes medidas para las modalidades de Gimnasia Artística y Gimnasia Rítmica

\begin{tabular}{|c|c|c|c|c|}
\hline & \multicolumn{2}{|c|}{ Gimnasia Artística } & \multicolumn{2}{|c|}{ Gimnasia Rítmica } \\
\hline & Media & \pm & Media & \pm \\
\hline Peso & 30.19 & 9.10 & 36.29 & 7.67 \\
\hline Altura & 1.38 & .18 & 1.45 & .11 \\
\hline Pliegue Biceps & 5.03 & 1.59 & 6.75 & 2.21 \\
\hline Pliegue Tríceps & 9.91 & 2.53 & 9.90 & 1.86 \\
\hline Pliegue Axilar & 5.63 & 1.67 & 6.38 & 1.40 \\
\hline Pliegue Pecho & 6.21 & 2.48 & 7.98 & 5.98 \\
\hline Pliegue Subescapular & 6.62 & 1.84 & 6.58 & 1.71 \\
\hline Pliegue Suprailiaco & 7.27 & 3.60 & 6.18 & 2.59 \\
\hline Pliegue Abdominal & 9.60 & 4.42 & 8.56 & 3.15 \\
\hline Pliegue Muslo & 14.26 & 3.49 & 15.92 & 3.48 \\
\hline Pliegue Gemelo & 14.27 & 5.05 & 14.16 & 2.82 \\
\hline Circunferencia Brazo & 19.84 & 3.06 & 19.91 & 1.63 \\
\hline Circunferencia Pecho & 65.11 & 7.29 & 68.62 & 6.57 \\
\hline Circunferencia Cintura & 57.76 & 5.57 & 58.66 & 4.72 \\
\hline Circunferencia Cadera & 69.03 & 9.75 & 70.73 & 20.93 \\
\hline Circunferencia Muslo & 39.34 & 5.75 & 41.58 & 4.10 \\
\hline Circunferencia Gemelo & 26.96 & 3.39 & 29.95 & 2.70 \\
\hline Diámetro Codo & 5.28 & .32 & 5.03 & .49 \\
\hline Diámetro Rodilla & 7.70 & .89 & 8.55 & .67 \\
\hline Test de Ruffier & 18.48 & 4.89 & 20.30 & 2.88 \\
\hline Altura Lumbar & 46.26 & 14.59 & 61.83 & 6.90 \\
\hline Flexibilidad Hombro & 58.50 & 12.26 & 24.00 & 22.38 \\
\hline Flexibilidad Isquiotibiales & 34.96 & 4.26 & 28.70 & 7.26 \\
\hline Flexibilidad Spagat & 54.07 & 16.99 & 35.58 & 17.56 \\
\hline Altura SJ & 21.19 & 4.18 & 23.25 & 2.80 \\
\hline Altura CJ & 24.27 & 4.27 & 24.40 & 3.47 \\
\hline Altura DJ & 11.45 & 4.72 & 9.32 & 3.55 \\
\hline Altura R5 & 2.64 & 1.36 & 2.85 & .84 \\
\hline$\%$ Graso & 23.28 & 6.84 & 20.35 & 4.67 \\
\hline IMC & 16.50 & 2.30 & 16.76 & 1.25 \\
\hline $\begin{array}{l}\text { Pico máximo de fuerza } \\
\text { Mano Derecha (MD) }\end{array}$ & 39.41 & 14.11 & 33.95 & 9.62 \\
\hline MIMF MD & .10 & .05 & .09 & .03 \\
\hline $\begin{array}{l}\text { Pico máximo de fuerza } \\
\text { Mano Izquierda (MI) }\end{array}$ & 38.63 & 13.30 & 30.82 & 7.84 \\
\hline MIMF MI & .12 & .06 & .07 & .01 \\
\hline $\begin{array}{l}\text { Pico máximo de fuerza Pie } \\
\text { Derecho (PD) }\end{array}$ & 479.81 & 179.82 & 514.29 & 146.22 \\
\hline MIMF PD & .60 & .39 & .70 & .26 \\
\hline $\begin{array}{l}\text { Pico máximo de fuerza Pie } \\
\text { Izquierdo (PI) }\end{array}$ & 504.11 & 143.70 & 549.41 & 143.28 \\
\hline MIMF PI & .74 & .39 & .77 & .30 \\
\hline
\end{tabular}

Existían dos anclajes a ambos lados del sujeto donde se sujetaban dos cadenas que convergían en el dinamómetro isométrico, el cual, en su otro extremo, se le acopló una pieza para encajar el pie de la gimnasta. De esta forma se quedaba sentada en el suelo con la espalda recta apoyada sobre la pared y las rodillas en total extensión. El pie, una vez encajado en la pieza del dinamómetro, formaba $90^{\circ}$ con la pierna. Ala señal, la gimnasta aplicaba la máxima fuerza posible realizando una extensión de tobillo durante 5 segundos (McGuigan \& Winchester, 2008). Tras finalizar, se procedía a la medición de la otra pierna (Figura 1). Se utilizó un módulo creado sobre el software LabView 8.5 que registraba los datos de carga mecánica, provenientes del dinamómetro, en tiempo real a una frecuencia de muestreo de $1000 \mathrm{~Hz}$. Estos datos se exportaban a un archivo de hoja de cálculo del software Excel 2007 de Microsoft ${ }^{\circledR}$ Office $^{(\mathrm{TM})}$. La conversión de la señal procedente del dinamómetro isométrico se realizó mediante la tarjeta de conversión analógico/digital y se elaboró un módulo sobre el software LabView 8.5 que registraba los datos de las diferentes variables de fuerza.

Y finalmente se llevaron a cabo las pruebas de flexibilidad que consistían en una extensión lumbar donde las participantes se tumbaban boca abajo sobre una plataforma acolchada y realizaban una extensión lumbar tomando como referencia el suelo, y se midió con una cinta métrica la distancia que había desde el suelo a la barbilla (Martínez, 2002); test de abductores mediante una posición de spagat anteroposterior tomando como referencia la pared, y se midió con una cinta métrica la distancia que había desde el tobillo a la pared (Moras, 1992); una flexión de tronco hacia delante con las piernas estiradas subidos a una silla de $40 \mathrm{~cm}$ de altura, y se midió con una cinta métrica la distancia que había desde el dedo corazón hasta el suelo (López, Ferragut, Alacid, Yuste \& García, 2008); y una prueba de flexibilidad de hombros utilizando una pica de un metro de longitud, cuyo fin fue agarrar la pica con las manos lo más juntas posible y con los codos estirados llevar los brazos desde delante a atrás del tronco, y se midió con una cinta métrica la distancia que había entre las dos manos (García, Navarro, \& Ruiz, 1996). Se decidió este orden de medición con el fin de que ninguna de las articulaciones se viera afectadas por una medición anterior.

\section{anter. \\ 2.4. Tratamiento estadístico}

El análisis estadístico fue realizado con el paquete estadístico Statistical Package for the Social Sciences 15.0. (@ 2008 SPSS Inc.).

Se realizó la prueba de normalidad Kolmogorov-Smirnov para determinar si nuestras variables de estudio eran normales, y la prueba de Levene para determinar si los datos de las mismas son homogéneos.

Además de realizar un análisis descriptivo de las variables, se realizó un análisis de correlaciones bivariadas en cada modalidad gimnástica para ver la relación particular que guardan las variables de estudio dentro de cada grupo, así como una Prueba $\mathrm{T}$ de medidas independientes para determinar las diferencias de medias entre las modalidades gimnásticas según las variables de análisis.

\section{Resultados}

Las pruebas de Kolmogorov-Smirnov confirmaron que las variables eran normales. La prueba de homogeneidad de varianza de Levene confirmó que todas las variables eras homogéneas.

En la Tabla 1, se muestran los análisis descriptivos de las variables dependientes medidas para las modalidades de GAF y GR.

En cuanto a las correlaciones bivariadas, destacan las correlaciones observadas entre el \% graso y el IMC, con el resto de variables. Específicamente, el grupo GR consiguió además una correlación positiva entre su IMC y el pliegue abdominal ( $\mathrm{r}=.652 ; p<.05)$; y entre su IMC y el diámetro del codo $(\mathrm{r}=.803 ; p<.01)$. Además, obtiene una relación negativa entre las variables \% graso y las variables mejor $\mathrm{SJ}(\mathrm{r}=-.774$; $p<.01)$, y mejor CMJ (r=-.600; $p<.05)$. Incluso, existió una relación negativa entre el \% graso y la variable MIMFmax del pie derecho (r=$\left..753^{*} ; p<.05\right)$. 


\begin{tabular}{|c|c|c|c|c|c|c|}
\hline VARIABLES & & mean & \pm & $D T$ & sig. & $\begin{array}{c}\begin{array}{c}\text { Diferencias de } \\
\text { medias }\end{array} \\
\end{array}$ \\
\hline \multirow{2}{*}{$\begin{array}{l}\text { Diámetro de rodilla } \\
(\mathrm{cm})\end{array}$} & GAF & 7.70 & \pm & .89 & \multirow[b]{2}{*}{.03} & \multirow{2}{*}{$-.85^{*}$} \\
\hline & GR & 8.55 & \pm & .67 & & \\
\hline \multirow{2}{*}{ Altura lumbar $(\mathrm{cm})$} & GAF & 50.08 & \pm & 8.08 & \multirow{2}{*}{.00} & \multirow{2}{*}{$11.76^{* *}$} \\
\hline & GR & 61.83 & \pm & 6.91 & & \\
\hline \multirow{2}{*}{$\begin{array}{c}\text { Flexibilidad } \\
\text { hombros (cm.) }\end{array}$} & GAF & 58.50 & \pm & 1226 & \multirow{2}{*}{.00} & \multirow{2}{*}{$34.50^{* *}$} \\
\hline & GR & 24.00 & \pm & 22.38 & & \\
\hline \multirow{2}{*}{$\begin{array}{c}\text { Flexibilidad } \\
\text { isquiotibiales }(\mathrm{cm} .)\end{array}$} & GAF & 34.96 & \pm & 4.27 & \multirow{2}{*}{.03} & \multirow{2}{*}{$6.25^{*}$} \\
\hline & GR & 28.71 & \pm & 7.27 & & \\
\hline \multirow{2}{*}{$\begin{array}{l}\text { Flexibilidad spagat } \\
\qquad \mathrm{cm})\end{array}$} & GAF & 54.08 & \pm & 16.99 & \multirow{2}{*}{.04} & \multirow{2}{*}{$18.49^{*}$} \\
\hline & GR & 35.58 & \pm & 1757 & & \\
\hline \multirow{2}{*}{$\begin{array}{c}\text { Pliegue del Bíceps } \\
(\mathrm{cm})\end{array}$} & GAF & 5.03 & \pm & 1.59 & \multirow{2}{*}{.036} & \multirow{2}{*}{$-1.71 *$} \\
\hline & GR & 6.75 & \pm & 2.21 & & \\
\hline \multirow{2}{*}{$\begin{array}{l}\text { Circunferen cia } \\
\text { Gemelo (cm.) }\end{array}$} & GAF & 26.96 & \pm & 3.39 & \multirow{2}{*}{0.23} & \multirow{2}{*}{$-2.99 *$} \\
\hline & GR & 29.95 & \pm & 2.70 & & \\
\hline${ }^{* * *} \mathrm{p}<.01, * \mathrm{p}<.05$ & & & & & & \\
\hline
\end{tabular}

Por otro lado, el grupo de GR obtiene una relación negativa entre el porcentaje graso y la altura conseguida en los saltos SJ y CJ. Esta relación encuentra justificación en el hecho de que un deportista que busca desplazarse en contra de la gravedad, cuanto menores niveles de grasa mejor, ya que la grasa actúa como un peso inerte (Esparza, 1993).

Pérez et al. (2006) observaron que a una edad tan temprana como los 10 años, en niñas que practicaban gimnasia rítmica no se producía correlación entre la masa magra total y la altura del vuelo alcanzado tanto en el salto SJ como en el salto CMJ, pero sí con el porcentaje graso ya que mostraba una correlación negativa con el rendimiento en el salto vertical, al igual que en nuestroestudio, donde se encontró una correlación negativa entre el porcentaje graso y el salto SJ, en GR. Igualmente, Sabido (2008) obtuvo relaciones similares entre ambas variables pero en la modalidad de gimnasia artística, lo que demuestra la importancia de tener niveles bajos de porcentaje graso a la hora de alcanzar valores destacables en la capacidad de salto.

Continuando con la modalidad de GR, el porcentaje graso

En cambio, el grupo GAF tuvo una relación positiva entre su IMC y el pliegue del tríceps $\left(\mathrm{r}=.796^{* *} ; p<.01\right)$, pliegue $\operatorname{axilar}\left(\mathrm{r}=.879^{* *} ; p<.01\right)$, pliegue suprailiaco $\left(\mathrm{r}=.932^{* *} ; p<.01\right)$ y pliegue del muslo $\left(\mathrm{r}=.735^{* *}\right.$; $p<.01)$; así como una relación negativa entre su IMC y la flexibilidad de isquiotibiales $\left(\mathrm{r}=-.729^{* *} ; p<.01\right)$.

Sin embargo, en el análisis de Prueba $T$ de medidas independientes, se observaron diferencias significativas entre ambos grupos en determinadas variables (Tabla 2). Por ejemplo, el grupo GAF obtuvo mayores niveles de flexibilidad en hombro $(\mathrm{T}(1.23)=4.83 ; \mathrm{p}<.001)$, isquiotibiales $(\mathrm{T}(1.23)=2.64 ; p<.05)$ y spagat $(\mathrm{T}(1.23)=2.67 ; \mathrm{p}<.05)$. En cambio, el grupo GR presentó mayor pliegue del bíceps (T(1.23)= $2.23 ; p<.05)$, circunferencia del gemelo $(\mathrm{T}(1.23)=2.42 ; p<.05)$, diámetro de la rodilla $(\mathrm{T}(1.23)=2.66 ; p<.05)$; y flexibilidad lumbar $(\mathrm{T}(1.23)=$ $3.89 ; p<.01)$. No se encontraron diferencias significativas entre ambas modalidades, para el resto de variables dependientes medidas.

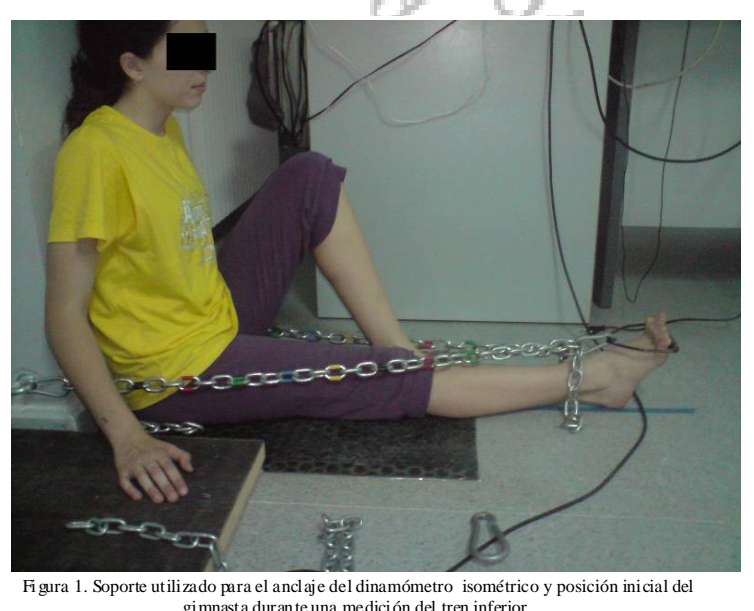

imnasta durante una medición del tren inferior.

\section{Discusión}

La relación encontrada en la muestra de gimnastas entre el IMC y diversas variables antropométricas también se verificó en el estudio de Albala, Kain, Burrows y Díaz (2000) quienes determinaron que el IMC y los pliegues corporales van asociados; cuestión que permite determinar valores como el porcentaje graso, incluso la obesidad en general. Recientemente, Cossio-Bolanos, Arruda y De Marco (2010) concluyeron la relación existente entre el IMC y las circunferencias corporales del brazo, el muslo y la pantorrilla; así como en la tesis de Martínez (2010), donde se determinó una correlación positiva del IMC con los pliegues cutáneos y con las circunferencias corporales.

La correlación negativa encontrada en la modalidad de GAF, entre la flexibilidad de isquiotibiales y el IMC, no se encuentra en el estudio de Silva y Bonorino (2008) ya que no encontraron correlaciones entre ambas variables. Esto puede ser debido a la diferencia de edad entre las participantes del grupo GAF, lo cual influiría en el IMC de las mismas.

correlacionó también negativamente con la variable máximo índice de manifestación de la fuerza en la pierna derecha. Este resultado está apoyado por Sancesario y Rosales (2006), en su estudio sobre las características antropométricas de luchadores, ya que consideraron que la grasa no constituye un elemento activo en la ejecución de los movimientos corporales y que más bien su aumento provoca una disminución de la fuerza del atleta. De forma complementaria, Marrodán et al. (2009) apoyaron este resultado al relacionar fuertemente la fuerza estática con el peso graso de manera negativa, determinando que el peso magro es el que realmente influye en la mayor o menor capacidad para generar fuerza. Y Martínez (2010) encontró la misma correlación en un grupo de escolares con edades comprendidas entre los 11 y los 13 años.

A nivel inferencial, en cuanto a las diferencias en las pruebas de flexibilidad, las gimnastas de la modalidad de GR tuvieron más flexibilidad lumbar debido al trabajo específico que realizan y presencia de arqueos en dicha modalidad gimnástica, aunque en GAF también hay trabajo de arqueos específicos, pero es en la modalidad de GR donde se trabaja más la flexibilidad hasta niveles máximos. De hecho, Zetaruk, Violan, Zurakowski, Mitchell y Micheli (2006) afirmaron que la flexibilidad lumbar es la más trabajada en la modalidad de gimnasia rítmica, y que es necesario compensar esta flexibilidad con trabajos de fuerza, con el fin de evitar la lumbalgia, muy acusada en esta modalidad. Sin embargo, los resultados obtenidos en pruebas como flexibilidad de hombros, de isquiotibiales y spagat mostraron mejores valores para la modalidad de GAF. Este comportamiento puede estar motivado por el hecho de que en esta modalidad gimnástica se realizan trabajos específicos de flexibilidad tanto de hombros como de isquiotibiales, necesarios para ejecutar tanto estéticamente como correctamente los elementos en aparatos como las paralelas y la barra de equilibrios. La mayoría de los elementos como stadler en paralelas o palomas en barra de equilibrios, precisan de un trabajo muy específico de fortalecimiento y flexibilidad en isquiotibiales. También, la flexibilidad de hombros se hace necesaria para la correcta ejecución de molinos en paralelas y flic-flac en barra de equilibrios. Según León (2006), la flexibilidad es el aspecto más significativo de esta modalidad deportiva, ya que es la que te permite alcanzar grandes rangos de movimientos, que junto a la fuerza permiten realizar de manera eficiente los elementos exigidos en la gimnasia de élite. Además, Faria y Faria (1989) determinaron que una de las características de un gimnasta élite, son unos niveles de movilidad elevados principalmente en la articulación de la cadera y del hombro, característica que sigue siendo muy importante en la gimnasia de élite actual.

No encontramos diferencias significativas entre las dos modalidades gimnásticas en los saltos SJ, CMJ, DJ y R5, al igual que en el estudio de Grande, et al., (2009), que no encontraron diferencias significativas entre GAF y GR, sin embargo afirmaron que las gimnastas de GAF son las que mayores alturas de salto presentan, tanto en el SJ como en el CMJ. Hecho que no apoya los análisis descriptivos observados en el presente estudio, en los cuales observamos que la modalidad que presenta mayor altura en los saltos SJ y CMJ, son las participantes de GR. Esto se debe a que la edad de las gimnastas de la modalidad de GR es mayor, 
con lo cual llevan más años de entrenamiento y esto les proporciona una mayor altura de salto, así como una mayor fuerza de ambas piernas.

\section{Conclusiones}

Este estudio ha encontrado diferencias en composición corporal y pruebas físicas en gimnastas de modalidades de rítmica y artística además de encontrar relaciones entre el rendimiento de dichas pruebas y variables antropométricas. Estos resultados permiten conocer mejor las características de las gimnastas de tecnificación de estas modalidades, aunque estudios longitudinales serían convenientes introducir a fin de observar cómo evolucionan y se relacionan dichas variables en cada modalidad gimnástica a lo largo de ciclos competitivos o deportivos. En relación con los objetivos planteados, podemos concluir que las pruebas más útiles para la predicción del rendimiento en las modalidades gimnásticas son aquellas relacionadas con el salto, la fuerza isométrica, la flexibilidad y las características antropométricas.

Por tanto, los resultados obtenidos permiten conocer unos primeros valores referenciales en variables determinantes de condición física, en gimnastas femeninas de tecnificación. El análisis de estos valores debería ser el punto de partida a la preparación de entrenamientos más específicos. Más adelante, sería oportuno relacionar la evolución de dichos valores de condición física con el resultado deportivo alcanzado en situación de competición

\section{Agradecimientos}

Agradecemos a la Federación Extremeña de Gimnasia su colaboración en la gestión de esta investigación, así como a las gimnastas y sus padres en la participación y colaboración en el desarrollo de las sesiones de mediciones.

\section{Referencias}

Albala, C., Kain, J., Burrows, R., \& Díaz, E. (2000). Obesidad: Un desafío pendiente. Monografía. Santiago: Universitaria.

Ariza, J. C. (2004). La fuerza relativa como variable de pronosticación del rendimiento deportivo en gimnasia artística. Kronos, Volumen III, 6, 60-73.

Bosco, C. (1994). La valoración de la fuerza con el test de Bosco. Barcelona: Paidotribo.

Bradshaw, E., \& Le Rossignol, P. (2004). Anthropometric and biomechanical field measures of floor and vault ability in 8 to 14 years old talent-selected gymnasts. Sports Biomechanics, 3, 249-262.

Cossio-bolanos, M, Arruda, M., \& De Marco, A. (2010). Correlación entre el índice de masa corporal y las circunferencias corporales de niños de 4 a 10 años. Anales de la Facultad de Medicina, 71(2), 79-82.

Deutz, R. C., Benardot, D., Martin, D. E., \& Cody, M. M. (2000). Relationship between energy deficits and body composition in eite female gymnasts and runners. Medicine and Science in Sports Exercise, 32(3), 659-668.

Díaz, M., Mauri, E., García, Y., \& Jiménez, C. (2008). Perfil antropométrico comparativo de la selección nacional de gimnasia artística femenina (2008) y el perfil del campeonato mundial en Rótterdam, Holanda (1987). EF Deportes.com, Revista Digital, año 13, 123. Recuperado el día 25 de Mayo del 2011.

Esparza, F. (1993). Manual de Cineantropometría. Monografías Femede. Grupo Español de Cineantropometría (GREC). Madrid: Femede.

Faria, I. E., \& Faria, E. W. (1989). Relationship of the anthropometric and physical characteristics of male junior gymnasts to performance. Journal of Sport and Medical Physical Fitness, 29(4):369-378

Fernández, J. C., Reina, A., Muñoz, R., \& Ruiz, A. (2005). ¿Influye el tipo de entrenamiento de la amplitud de movimiento en el salto en mujeres? http:// www.efdeportes.com/. Revista Digital - Buenos Aires - Año 10 - № 83 - Abril de 2005.

García, E., Navarro, A., \& Navarro, J. M. (2009). Test más apropiados para la valoración funcional del deportista en gimnasia rítmica. EF Deportes.om, Revista Digital, año 13, 129. Recuperado el día 3 de Marzo del 2011.

García Manso, J. M., Navarro Valdivieso, M., \& Ruiz Caballero, J. A. (1996). Pruebas para la valoración de la capacidad motriz en el deporte. Evaluación de la condición física. Madrid, Gymnos.

González, J. J., \& Gorostiaga, E. (2002). Fundamentos del Entrenamiento de la Fuerza. Barcelona. Ed. Inde, edición 3 .

Grande, I., Figueroa, J., Hontoria, M., \& Bautista,A. (2009). Evolución y comparación de la capacidad de salto de los equipos nacionales de gimnasia artística femenina y rítmica durante la preparación del Campeonato del Mundo 2007. Kronos, 7(14), 91-94.

Grande, I., Sampedro, J., Rivilla-García, J., Bofill, A., \& Hontoria, M. (2010). Evolución y relación de la capacidad de salto y amortiguación en gimnastas de rítmica de alto nivel. Cuadernos de Psicología del Deporte, 10: 43-50.

Irurtia Amigo, A., Busquets Faciabén, A., Marina Evrard, M., Galilea Ballarini, P. A., \& Carrasco Marginet, M. (2009). Talla, peso, somatotipo y composición corporal en gimnastas de elite españoles desde la infancia hasta la edad adulta. Apunts, 44(161), 18-28.

Katch, F. I., \& McArdle, W. D. (1975). Validity of body composition prediction equations for college men and women. The American Journal of Clinical Nutrition, 28(2):105-109.

León, J. (2006). Estudio del uso de test físicos, psicológicos y fisiológicos para estimar el estado de rendimiento de la selección nacional de Gimnasia Artística Masculina. Tesis doctoral no publicada, Universidad Pablode Olavide. Sevilla.

López Bedoya, J., Vernetta, M., \& De La Cruz, J. C. (1993). Características morfológicas y proceso de maduración de las gimnastas de alto nivel. Archivos de Medicina del Deporte, 10(37), 49-55.

López Miñarro, P. A., Ferragut Fiol, C., Alacid Cárceles, F., Yuste Lucasa, J. L., \& García Ibarra, A. (2008). Validez de los test dedos-planta y dedos-suelo para la valoración de la extensibilidad isquiosural en piragüistas de categoría infantil. Apunts medicina del esport, 157, 24-29.

Márquez, F. J., Orihuelo, A., Jiménez, J., \& Fernández, J. C. (2005). Efectos de la fatiga muscular sobre la fuerza explosiva en el squat jump y la velocidad. www.efdeportes.com. Revista Digital- Buenos Aires-Año 10- No 88- Septiembre de 2005.

Marrodán, M. D., Romero, J. F., Moreno, S., Mesa, M. S., Cabañas, M. D., Pacheco, J. L., \& González, M. (2009). Handgrip strength in children and teenagers aged from 6 to 18 years: Reference values and relationship with size and body composition. Anales de pediatría, 70(4), 340-348.

Martínez, E. (2002). Pruebas de aptitud física. Barcelona, Paidotribo.

Martínez Castañeda, R. (2010). Valoración de la condición física en relación con la salud en escolares preadolescentes de la provincia de León: influencia de la actividad física en el sobrepeso, la obesidad y el riesgo de síndrome metabólico. Tesis Doctoral. Universidad de León, León.

McGuigan, M., \& Winchester, J. (2008). The relationship between isometric and dynamic strength in college football players. Journal of Sports Science and Medicine, 7, 101-105.

Mendizábal, S. (2001). Fundamentos de la Gimnasia Rítmica. Mitos y realidades. Ed. Gymnos. Madrid.

Moras, G (1992). Análisis crítico de los actuales tests de flexibilidad. Correlación 1 entre algunos de los tests actuales y diversas medidas antropométricas. Apunts, 29, 127-137.

Morenilla, L., Sierra, E.; Bueno, I., \& Rodríguez, A. (2001). Estudio comparativo sobre las manifestaciones de la fuerza de salto en gimnastas Gallegos de modalidades diferentes. ISimposium Internacional de Actividades Gimnásticas y Acrobáticas (VII Simposium Nacional). Enseñanza y Entrenamiento de la Gimnasia y la Acrobacia INEF Galicia, Universidade da Coruña, España.

Pérez-Gómez, J., Vicente-Rodríguez, G, Ara, I., Arteaga, R., Calbet, J. A. L., \& Dorado, C. (2006). Capacidad de salto en niñas prepúberes que practican gimnasia rítmica. Motricidad, Journal of Human Movement, 15, 273-286.

Sabido, R. (2008). Elaboración de programas de entrenamiento para la mejora del salto vertical en gimnasia artística. Tesis doctoral no publicada. Universidad de Extremadura.

Sancesario, L. A. \& Rosales, A. R. (2006). Valores antropométricos de referencia para la clasificación del talento en la lucha. Revista Internacional de Medicina y Ciencias de la Actividad Física y el Deporte, 6(24), 248-254.

Sands, W. A., Caine, D. J., \& Borms, J. (2003). Scientific Aspects of Women`s Gymnastics. Medicines and Sport Science, 45, 46-71.

Silva, A. H., \& Bonorino, K. C. (2008). Índice de masa corporal y flexibilidad de los bailarines de la danza contemporánea y del ballet clásico. Fittness Performance Journal, 7(1), 48-51.

Silva, M. R. (2005). Composición corporal de las gimnastas de competición. http:/ /www.efdeportes.com/, Revista Digital-Buenos Aires-Año 10-N ${ }^{\circ}$-Junio de 2005.

Taaffe, D. R., Robinson, T. L., Snow, C. M., \& Marcus, R. (1997). High-Impact Exercise Promotes Bone Gain in Well-Trained Female Athletes. Journal of Bone and Mineral Research, 12(2), 255-260.

Weimann, E. (2002). Gender-related differences in elite gymnasts: the female athlete triad. Journal of Applied Physiology, 92, 2146-2152.

Weimann, E., Witzel, C., Schwidergall, S., \& Böhles, H. J. (2000). Peripubertal perturbations in eite gymnasts caused by sport specific training regimes and inadequate nutritional intake. International Journal of Sports Medicine, 21, 210-215.

Yuhasz, M. S. (1974). Physical Fitness Manual. London Ontario, University of Western Ontario.

Zetaruk, M., Violan, M., Zurakowski, D., Mitchell, W., \& Micheli, L. Recomendaciones para el entrenamiento y prevención de lesiones en gimnastas de rítmica de elite. Apunts, 151: 100-106, 2006. 